\title{
Maternal antioxidant intake during pregnancy and the development of cow's milk allergy in the offspring
}

Jetta Tuokkola ${ }^{1,2 *}$, Anni Lamminsalo ${ }^{3 *}$, Johanna Metsälä², Hanna-Mari Takkinen², Heli Tapanainen², Mari Åkerlund ${ }^{2,3}$, Sari Niinistö ${ }^{2}$, Jorma Toppari ${ }^{4}$, Jorma Ilonen ${ }^{5,6}$, Riitta Veijola7, Mikael Knip ${ }^{1,8,9,10}$, Minna Kaila ${ }^{11,12 * *}$, Suvi M Virtanen²,3,10,13**

*Jetta Tuokkola and Anni Lamminsalo contributed equally to the study

** Minna Kaila and Suvi Virtanen contributed equally to the study

${ }^{1}$ New Children's Hospital and Clinical Nutrition Unit, Internal Medicine and Rehabilitation, University of Helsinki, Helsinki University Hospital, 00290 Helsinki, Finland

${ }^{2}$ Public Health Promotion Unit, Finnish institute for health and welfare, 00271 Helsinki, Finland ${ }^{3}$ Faculty of Social Sciences/Health Sciences, Tampere University, 33014, Tampere, Finland

${ }^{4}$ Department of Pediatrics, University of Turku, 20014, Turku, Finland

${ }^{5}$ Department of Clinical Microbiology, University of Eastern Finland, 70211, Kuopio, Finland

${ }^{6}$ Immunogenetics Laboratory, University of Turku, 20014, Turku, Finland

${ }^{7}$ Department of Paediatrics, University of Oulu, 90014 Oulu, Finland

${ }^{8}$ Research Program for Clinical and Molecular Metabolism, Faculty of Medicine, University of Helsinki, 00014 Helsinki, Finland

${ }^{9}$ Folkhälsan Research Institute, 00251, Helsinki, Finland

${ }^{10}$ Center for Child Health Research, Tampere University, Tampere University Hospital, 33521, Tampere, Finland

${ }^{11}$ Public Health Medicine, University of Helsinki, Helsinki University Hospital, 00014, Helsinki, Finland

${ }^{12}$ Department of Paediatrics, Tampere University Hospital, 33521, Tampere, Finland

${ }^{13}$ Research, Development and Innovation Center, Tampere University Hospital, 33521 Tampere, Finland

Corresponding author: Anni Lamminsalo, anni.lamminsalo@tuni.fi, +358407323184, Faculty of Social Sciences, Unit of Health Sciences, 33014 Tampere University, Tampere, Finland

Running head: Maternal antioxidant intake and child's CMA key words: antioxidants, cow's milk allergy, maternal diet, pregnancy 
Abstract

Cow's milk allergy (CMA) is the most common food allergy in young children and it is often the first manifestation of atopic diseases. Accordingly, very early environmental factors, such as maternal diet during pregnancy, may play a role in the development of CMA, but the evidence is limited. The aim of this study was to investigate the association between maternal intake of antioxidant nutrients during pregnancy and the subsequent development of CMA in the offspring in a prospective, population-based birth cohort within the Finnish Type 1 Diabetes Prediction and Prevention Study. Maternal dietary information during pregnancy was collected with a detailed, validated food frequency questionnaire. The maternal dietary information and the information on putative confounding factors was available for 4403 children. Information on diagnosed CMA ( $n=448)$, was obtained from a medical registry and queried from the parents up to child's age of 3 years. The Finnish food composition database was used to calculate the average daily intake of nutrients. Logistic regression was applied for statistical analyses, and the nutrient intakes were adjusted for energy intake. Odds ratios are presented per one standard deviation increment of the particular nutrient intake. Maternal total and dietary intake of betacarotene was associated with an increased risk of CMA in the offspring when adjusted for the putative confounding factors (total: OR 1.10 95\% CI 1.02-1.20, dietary: OR 1.10 95\% CI 1.011.19). Using dietary supplements containing antioxidants in addition to a balanced diet may not confer any additional benefits. 
Introduction

Cow's milk allergy (CMA) is a common food allergy, affecting 2-6\% of Finnish children under the age of 3-4 years ${ }^{(1,2)}$. It is often the first manifestation of allergic diseases. Maternal nutrition during pregnancy, among other environmental factors has been implicated to play a role on the development of allergic diseases in the offspring ${ }^{(3)}$. Because CMA usually manifests during infancy, these early exposures may be of importance in the development of CMA. Antioxidant nutrients have been shown to exert immunologic effects, and they could potentially influence the development of allergic diseases ${ }^{(4)}$. Two diverse hypothesis have emerged: epidemiological evidence suggests that a diet lower in antioxidants is associated with an increase in allergic diseases $^{(5)}$, whereas the mechanistic hypothesis points towards antioxidants leading to suppression of T helper type 1 (Th1) cytokines and thus a higher susceptibility to allergic diseases (reviewed by Allan et al. ${ }^{(6)}$ ). In addition to antioxidative effects, the nutrients may have other immunoregulatory pathways. For example, retinoic acid is involved in regulatory T-cell formation and thus the development of oral tolerance ${ }^{(7)}$. The most recent meta-analysis on maternal nutrition during pregnancy and allergic diseases in the child states that the current evidence is in favor of protective association between vitamin $\mathrm{E}$ as well as for zinc and childhood wheezing, but is inconclusive against other allergic diseases. For other antioxidant nutrients the associations were even more contradictory ${ }^{(8)}$.

The knowledge of maternal dietary factors affecting the risk of CMA in the offspring is limited. One study reported that maternal intake of vitamin D from the diet during pregnancy was associated with decreased risk and intake of folate with increased risk of CMA in the offspring ${ }^{(9)}$. To our knowledge, associations between antioxidant nutrient intake during pregnancy and the development of CMA in the offspring have not been reported before. The aim of this study was to investigate the associations between maternal intake of antioxidant vitamins and minerals during pregnancy both from diet and supplements and the subsequent development of CMA in the offspring. 
Subjects and methods

\section{Subjects}

The Finnish Type 1 Diabetes Prediction and Prevention (DIPP) Study is a multidisciplinary prospective population-based birth cohort study ${ }^{(10)}$. The study is conducted in three university hospitals in Finland (Turku, Oulu, and Tampere) and after parental informed consent, all newborn infants from these areas were screened for HLA-conferred susceptibility to type 1 diabetes from cord blood samples. Infants who carry HLA genotypes conferring high and moderate risk for type 1 diabetes (14\% of those screened) were invited to participate in the study. The children with severe congenital abnormalities or diseases, or whose parents were of nonCaucasian origin or did not understand Finnish, Swedish or English were excluded. The study was conducted in accordance to the Declaration of Helsinki. The local Ethical Committees approved the study. All families have given their written informed consent.

The DIPP Nutrition study is a part of the main DIPP study comprising children born in the Oulu and Tampere areas. The present study comprised of 6288 children born between October 1997 and September 2004. Both maternal dietary data during pregnancy and the information of child's CMA was available for 4921 children (78•3\%) from a total of 4861 pregnancies. Complete information on background factors used in the current analyses in the pregnancy cohort was available for 4403 children (Figure 1).

When the children were 5 years of age, an Asthma and Allergy sub study (including parental history of allergy and child's animal contacts) was performed. All children at follow-up, also those who did not have maternal dietary data were invited. Of the 4075 children (65\% of the 6288 children) still at follow-up at 5 years, 3781 children (93\% of those 4075 invited) participated in the Asthma and Allergy sub study. Of these children, 2327 children had information also on maternal diet, basic background factors, and cow’s milk allergy, enabling a sub group analysis with information on parental allergies included in the current study. The flow chart of the cohort with maternal dietary data and the information of child's CMA is presented in Figure 1.

\section{Dietary assessment}

Maternal diet during pregnancy ( $8^{\text {th }}$ month) was assessed by a 181 -item semi-quantitative food frequency questionnaire (FFQ), which has been validated against food records (two times 5-day food record) in a setting that reflects the present study ${ }^{(11)}$. The validation study did not take into account the nutrient intake from supplements. In the validation study, Pearson correlations with 
food records were 0.37 for vitamin A, 0.71 for retinol, 0.53 for $\beta$-carotene, 0.22 for vitamin $\mathrm{E}$, 0.65 for vitamin C, 0.45 for zinc, and 0.46 for selenium ${ }^{(11)}$. The FFQ was designed to represent the entire diet over the $8^{\text {th }}$ pregnancy month. As in Finland mother's pregnancy leave begins right after $8^{\text {th }}$ pregnancy month, it is likely to be the most representative month of the average nutrient intake during the whole pregnancy. The mother's received the FFQ via mail after delivery, and they returned it at the 3-month study visit.

A trained study nurse checked the FFQ when returned. The FFQ was specifically designed to reflect Finnish food consumption habits and it assessed the consumption frequency of foods or food groups (not at all, number of times per day, week or month) as common serving sizes, such as a glass, a plateful or deciliter. The individual habits of fat used in cooking and baking were taken into account. The food consumption data were double entered. The FFQ was rejected if there were 10 or more missing frequencies or the form was inadequately filled in ( $n=53,1 \cdot 1 \%)$. Daily intakes of vitamins A, C, and E, $\beta$-carotene, retinol, selenium, and zinc were calculated with the use of the Finnish food composition database, Fineli ${ }^{\circledR},{ }^{(12)}$ by an in-house software of the Finnish institute for health and welfare. The detailed content of the FFQ and data processing have been described elsewhere ${ }^{(11,13)}$. During the study time the recipe compositions were updated in order to reflect the changes in food consumption habits and changes in the food market. That is why two versions of the database were used: the first version for the study years 1997-2002, and the second version for the years 2003-2004. The changes in recipes were mainly based on food consumption information of women aged 25-44 years from the national dietary surveys, FINDIET $1997^{(14)}$ and FINDIET $2002^{(15)}$. Recording of the FFQ and the accuracy of the nutrient database of the Finnish institute for health and welfare were checked at dietary analyses. The FFQ included also a question about dietary supplements, asking about the type, brand name, and manufacture's name, as well as the amount of each supplement per day or per week, and the pregnancy weeks during which the supplements were used. The nutrient contents of the dietary supplements were obtained from the National Food Administration, manufacturers, and from the Finnish pharmacopeia for supplements registered as drugs.

\section{Endpoints}

Information on CMA, obtained from the registers of the Social Insurance Institution, complemented with parental reports, was used as the endpoint. The register-based information on CMA was based on a granted special reimbursement for the costs of special infant formulas needed in the management of diagnosed CMA (ICD-10 codes L27.2 or K52.2). The special reimbursement is entitled to all Finnish infants up to age 2 years irrespective of infants/parents 
socioeconomic status, place of residence or place of treatment. An application and a certificate from a pediatrician, stating that the CMA diagnosis has been made according to specified medical criteria is required. Pediatricians have agreed on the criteria for the diagnosis, which, during the study period, was usually a response to an elimination diet and an open challenge, and rarely a response to an elimination diet with a positive skin prick test or a specific IgE. Only in some rare cases a double-blind placebo-controlled challenge was performed. In addition, CMA of the children was queried with open questions from parents at the age of 6 months and 1 and 2 years, and with a structured, validated questionnaire at the age of 3 years ${ }^{(16,17)}$.

\section{Background factors}

Families were asked for information on maternal and paternal vocational education, age, and place of residence at the recruitment. Information on pregnancy and delivery complications, gestational age, birth weight and height, earlier deliveries, and maternal smoking during pregnancy were received from the medical birth records of the delivery hospitals. Information about breastfeeding was asked from the parents in the dietary questionnaires. Among those families who participated in the Asthma and Allergy study at the child's age of 5 years, the parents were asked for their asthma and allergic rhinitis background, and child's animal contacts during the first year of life ${ }^{(18)}$.

\section{Statistical methods}

The differences in background factors between children with and without CMA were analyzed by using $\chi^{2}$-test. Logistic regression was applied to study the associations between maternal antioxidant nutrient intakes and the risk of CMA in the offspring. The possible reliance among siblings was accounted for by using the generalized estimating equations (GEE) with the sandwich estimator of variance to estimate regression coefficients in logistic regression analysis $^{(19)}$. Selection of variables included in adjusted models was based on previous evidence $^{(20-24)}$ as well as their association with CMA in the present study. The nutrient intakes were adjusted for energy intake by the residual method ${ }^{(25)}$ after logarithmic transformation. The total intake of each nutrient was calculated as the sum of intake from foods and supplements. The intake of nutrients from food alone and from food and supplements together were analyzed if the nutrient intake from supplements was meaningful. Nutrient intake variables were used as continuous explanatory variables in the analyses and first, the unadjusted analysis was conducted. The variables included in the first adjusted model were study center, sex, birth weight of the child, maternal age and education, maternal smoking during pregnancy, duration of 
gestation, mode of delivery, number of older siblings, season of birth, urbanity of living environment, and length of breastfeeding. These analyses were considered as main analyses. In addition we made analysis in a sub group of children who participated in the Asthma and Allergy study at the child age of 5 years. In this analysis the adjusted model included all those variables included in the main adjusted model as well as the information about maternal history of allergy, paternal history of allergy, visits to a stable and pet keeping during the child's first year of life. Due to the significant drop out of children at the follow-up at 5 years of age, when the Asthma and Allergy sub study was performed, we also repeated the main analyses in this sub group to evaluate whether the results, adjusted for allergy variables, are due to the reduced number of subjects or confounding by the allergy variables. Interaction between maternal history of allergy (mother has allergic rhinitis or asthma) and nutrient intakes were tested, and analyses were done separately for mothers with history of allergy and mothers without history of allergy if the interaction was significant $(\mathrm{p}<0 \cdot 05)$. SAS version $9 \cdot 3$ (SAS Institute Inc., Cary, NC, USA) was used in the analysis.

Results

The mean total and dietary intake of the antioxidant nutrients are shown in Table 1 . The cumulative incidence of CMA was $9 \cdot 3 \%$ by the age of 3 years. The background factors associated with an increased risk of CMA were male sex, high parental education level, parental allergic history and maternal use of vitamin supplements containing vitamin A, C and E during pregnancy (Table 2). Maternal smoking and having pets inside home during the child's $1^{\text {st }}$ year of life were, in turn, associated with a decreased risk of CMA.

Maternal total intake of $\beta$-carotene and intake of $\beta$-carotene and vitamin $E$ from food were associated with an increased risk of CMA in the offspring in the unadjusted model (Table 3). In the main analysis, after adjusting for putative baseline confounders, the associations remained for intake of $\beta$-carotene, both total (OR 1.10 95\% CI 1.02-1.20) and from food (OR 1.10 95\% CI 1.01-1.19) (Table 3).

In the sub group analysis, after adjusting for putative baseline confounders, parental history of allergy, and child's animal contacts during the first year of life, the intake of selenium from food (OR 0.85 95\% CI 0.74-0.98) was associated with a decreased risk, and the total intake of zinc (OR 1.13 95\% CI 1.00-1.27) and $\beta$-carotene (OR 1.12 95\% CI 1.00-1.26) with an increased risk of CMA in the 
offspring. These associations were observed also when adjusted only for putative baseline confounders (Supplementary 1).

An interaction between maternal history of allergy and the intake of antioxidant nutrients was observed for the total intake of vitamin $\mathrm{E}$ ( $\mathrm{p}$ for interaction 0.013 ) when adjusted for all the potential confounding factors, including paternal history of allergy. The total intake of vitamin $\mathrm{E}$ was associated with an increased risk of CMA in mothers with a history of allergy (OR 1.61 95\% CI 1.14-2.28), but not in mothers without such a history (OR 0.98 95\% CI 0.85-1.12). For other nutrients, no interactions with maternal allergic history were observed (data not shown).

\section{Discussion}

In this large population based birth cohort study we observed that maternal total and dietary intake of $\beta$-carotene during pregnancy were associated with an increased risk of CMA in the offspring up to child's age of 3 years.

A major strength of our study is that the data was collected prospectively from a large number of mothers and children. Furthermore, a major advantage is the good quality of dietary information, which was gathered by a detailed, validated FFQ. Cross-classification in quintiles by food consumption and nutrient intake was acceptable for all nutrients.

The sub group analysis, done within children participating in the Asthma and Allergy study, enabled us to take into account the parental history of allergy and the child's animal contacts during the first year of life as potential confounding factors. In this sub group analysis we did not observe difference between the results when the adjustment was made only for putative confounding factors and when further adjusted for parental history of allergy and the child's animal contacts during the first year of life. This suggests that these factors do not have a significant confounding role in our study group. However, the difference observed between the main and the sub group analyses suggests that the drop out had distorted the sub group results. A limitation of the present study is that the study subjects were selected based on HLA-conferred susceptibility to type 1 diabetes, representing about $14 \%$ of all newborn infants in Finland. These infants may have an increased intestinal permeability ${ }^{(27)}$, which may explain why the incidence of CMA in our study was higher than previously reported in Finland ${ }^{(1)}$. Therefore our results may not be fully generalizable to the unselected pediatric population. Estimating the nutrient intake by the FFQ has some limitations. First, as the mothers received the FFQ after delivery and were asked to retrospectively report their diet during the $8^{\text {th }}$ month of pregnancy, it is open for a recall bias. However, in the validation study the FFQ filled after delivery was considered to be 
equally representative of the diet during the $8^{\text {th }}$ month of pregnancy as FFQ filled during the $8^{\text {th }}$ month of pregnancy, therefore this recall bias should be minor. Second, the FFQ is reported to slightly overestimate the nutrient intake, however, the adjustment of nutrient intakes for energy intake should diminish this problem ${ }^{(11)}$. Third, as nutrient intake from supplements was not calculated in the validation study, the validity of the intake from supplements in the present study remains unclear. Fourth, in the validation study the Pearson correlation coefficient for Vitamin E was low ( $\mathrm{r}=0 \cdot 22)$, and therefore the observed associations for vitamin $\mathrm{E}$ might be diminished and should be interpreted cautiously. We took several possible confounders into account in order to specifically study the effects of the selected antioxidant nutrients, to avoid the bias in results from some other lifestyle factors, which are associated with the intake of these nutrients. Unfortunately, the diet of the children themselves was not controlled for. However, because we examined a large number of dietary variables in our analyses, it is possible that the significant association may only be due to chance. A further limitation is that we only had information about the age at diagnosis, and the onset of symptoms of CMA was not known, although these two should coincide.

To our knowledge, this is the first study to examine the association between antioxidant nutrient intake during pregnancy and the development of CMA in the offspring. Only one previous study has used food allergy as an outcome when studying the associations of maternal antioxidant intake with offspring allergic diseases. The authors suggested that vitamin $C$ and copper could be associated with a reduced risk of food allergy, but for vitamin E, $\beta$-carotene, or zinc no association was seen $^{(28)}$. Our observation on the association between maternal total and dietary intake of $\beta$-carotene and the increased risk of CMA in the offspring is in line with some mechanistic studies ${ }^{(29,30)}$, but is not supported by epidemiological findings ${ }^{(31,32)}$.

Evidence from epidemiological studies on the influences of maternal antioxidant intake during pregnancy on allergic outcomes in childhood is scarce, studies reporting mostly protective or non-significant associations. The most recent meta-analysis observed a protective association only for the maternal intake of vitamin $\mathrm{E}$ and zinc during pregnancy and wheezing in the offspring $^{(8)}$. In addition, two previous studies have reported that high maternal plasma selenium concentration during pregnancy ${ }^{(31)}$ or in cord blood ${ }^{(32)}$ was associated with a decreased risk of wheezing in the offspring. However, wheezing is a condition different from CMA and may not always be of atopic origin. The effect of oxidative stress on the pathogenesis of pulmonary manifestations of atopic disease is likely to differ from that of CMA. On the other hand, mechanistic studies have implied a possible predisposing effect of antioxidant nutrients on 
allergic diseases. The anti-inflammatory benefits of the antioxidant nutrient may be lost with higher doses because of the too strong inflammatory suppression which could lead to Th1 suppression promoting Th2 responses associated with allergic diseases ${ }^{(29,30)}$ Murr et al. have suggested that increased antioxidant intake could suppress cytokines, namely interferon- $\gamma$, leading to Th1 differentiation ${ }^{(33)}$. This suppression would then, due to cross-regulation, promote the development of a Th2 phenotype. In addition, previous in vitro studies have shown that vitamin A and its derivatives may favor Th2 immune responses ${ }^{(34)}$.

In Finland, the mothers who use dietary supplements receive higher amounts of antioxidant nutrients from their diet, have a higher education and smoke less compared to mothers who do not use any supplements ${ }^{(35)}$. This may have affected our results as those mothers with overall healthier lifestyle may seek help for their child's symptoms more actively, which may increase the child's likelihood of receiving a CMA diagnosis. Even though some associations prevailed after adjusting for maternal education, it is possible that our finding on maternal antioxidant nutrient intake and higher risk of CMA in the offspring is not causal.

Because allergic diseases are strongly hereditary, we examined the associations between nutrients and CMA in children separately for mothers with and without a history of allergy. We observed that maternal intake of vitamin E during pregnancy was associated with a higher risk of CMA only in the offspring of mothers with a history of allergy. However, this observation might be affected by the selection bias and therefore needs to be interpreted cautiously. Even so, it is possible that the maternal allergy influences the immunological environment in utero, therefore the maternal allergy may modify the immunological effects of nutrients during pregnancy ${ }^{(36)}$. Further studies are needed to explore the significance as well as the immunological mechanisms behind this finding.

In conclusion, these results are the first to link maternal antioxidant nutrient intake during pregnancy with the development of CMA in the offspring. For majority of studied antioxidant nutrients, we did not observe an association between maternal intake and CMA in the offspring. Thus, the maternal use of supplements containing antioxidant nutrients during pregnancy do not seem to have any additional benefits. A sufficient and balanced intake of antioxidant nutrients is best achieved by adhering to the dietary guidelines for pregnant women. 
Acknowledgements: We are extremely grateful to all the families who took part in this study. We would also like to acknowledge the excellent collaboration of the DIPP research nurses, doctors, nutritionists and laboratory staff over the years.

Financial suport: The study was supported by the Academy of Finland (grants 63672,79685, 79686, 80846, 201988, 210632, 129492, 126813, 276475), the Finnish Paediatric Research Foundation, the Juho Vainio Foundation, the Yrjö Jahnsson Foundation, the Competitive Research Funding of the Tampere University Hospital (grants 9L035, 9M029, 9P017, 9P057, 9R012, 9R055, 9S015, 9S074, 9T072, 9U016, 9U065, 9V012), Medical Research Funds of Turku and Oulu University Hospitals, the European Foundation for the Study of Diabetes, the Juvenile Diabetes Research Foundation (grants 197032, 4-1998-274, 4-1999-731, 4-2001-435), the Novo Nordisk Foundation and EU Biomed 2 (BMH4-CT98-3314), Doctoral Programs for Public Health, Foundation for Allergy Research, Research Foundation of Orion Corporation, Tampere Tuberculosis Foundation, Päivikki and Sakari Sohlberg foundation and the Jalmari and Rauha Ahokas Foundation. None of the funders had any role in the design, analysis or writing of this article

The authors contributions: S.M.V., J.Tuokkola and M. Kaila were responsible for the current study design and concept. J.I., M.Knip, J.Toppari and R.V. are members of the stearing committee of the DIPP study. S.M.V. designed the DIPP nutrition study and within the DIPP nutrition study the allergy study was designed by S.M.V. and M. Kaila. J.Tuokkola and S.M.V. conducted research. H.-M.T. and H.T. were responsible for the statistical analysis. R.V. was responsible for the clinical work in Oulu, M. Knip was responsible of the clinical work in Tampere. J.Tuokkola and A.L. wrote the first version of the article with equal contribution. J.M., M. Kaila, and S.M.V. particitipated in the writing process. S.M.V and M. Kaila. had the primary responsibilty for the final work with equal contribution. All the authors particitipated in the critical revision of the manuscript and have accepted the final version.

The authors declare that there are no conflict of interest. 


\section{References}

1. Pyrhönen K, Näyh S, Kaila M et al. (2009) Occurrence of parent-reported food hypersensitivities and food allergies among children aged 1-4 yr. Pediatr Allergy Immunol. 20, 328-338

2. Saarinen KM, Juntunen-Backman K, Järvenpää AL et al. (1999) Supplementary feeding in maternity hospitals and the risk of cow's milk allergy: A prospective study of 6209 infants. J Allergy Clin Immunol 104, 457-61.

3. West C.E., D’Vaz N., Prescott S.L (2011). Dietary immunomodulatory factors in the development of immune tolerance. Curr. Allergy Asthma Rep. 11(4):325-333.

4. Sardecka, I., Krogulska, A., Toporowska-Kowalska, E. (2017). The influence of dietary immunomodulatory factors on development of food allergy in children. Postepyy Dermatol Alergol, 34(2), 89-96.

5. Miyake Y, Sasaki S, Tanaka K, et al. (2010). Consumption of vegetables, fruit, and antioxidants during pregnancy and wheeze and eczema in infants. Allergy 65, 758-65.

6. Allan K, Kelly FJ, Devereux G (2010) Antioxidants and allergic disease: a case of too little or too much? Clin Exp Allergy 40, 370-380.

7. Hall JA, Grainger JR, Spencer SP et al. (2011) The role of retinoic acid in tolerance and immunity. Immunity 35(1), 13-22.

8. Beckhaus AA, Garcia-Marcos L, Forno E et al. (2015). Maternal nutrition during pregnancy and risk of asthma, wheeze, and atopic diseases during childhood: a systematic review and metaanalysis. Allergy 70, 1588-1604.

9. Tuokkola, J., Luukkainen, P., Kaila, M. et al. (2016). Maternal dietary folate, folic acid and vitamin D intakes during pregnancy and lactation and the risk of cows' milk allergy in the offspring. Br J Nutr 116, 710-718.

10. Kupila A, Muona P, Simell T et al. (2001) Feasibility of genetic and immunological prediction of type I diabetes in a population-based birth cohort. Diabetologia 44, 290-297.

11. Erkkola M, Karppinen M, Javanainen J et al. (2001) Validity and reproducibility of a food frequency questionnaire for pregnant Finnish women. Am J Epidemiol 154, 466-476.

12. National Institute for Health and Welfare, Nutrition Unit (2009) National Institute for Health and Welfare (2009) Fineli ${ }^{\circledR}$ - Finnish Food Composition Database. Http://Www.Fineli.Fi. (accessed November 2019)

13. Prasad M, Lumia M, Erkkola M et al. (2010) Diet composition of pregnant Finnish women: changes over time and across seasons. Public Health Nutr 13, 939-946.

14. National Public Health Institute (1998) The 1997 Dietary Survey of Finnish Adults. Helsinki: Hakapaino Oy.

15. Männisto S, Ovaskainen M, Valsta L (2003) The National Findiet 2002 Study. Helsinki: Hakapaino Oy. 
16. Tuokkola J, Kaila M, Pietinen P et al. (2008) Agreement between parental reports and patient records in food allergies among infants and young children in Finland. $J$ Eval Clin Pract 14, 984-989.

17. Tuokkola J, Luukkainen P, Kaila M et al. (2010) Validation of a questionnaire on cow's milk allergy: parental reports and physicians diagnosis. Acta Paediatr 99, 1273-1275.

18. Nwaru BI, Lumia M, Kaila M et al. (2011) Validation of the Finnish ISAAC questionnaire on asthma against anti-asthmatic medication reimbursement database in 5-year-old children. Clin Respir J 5, 211218.

19. Liang KY \& Zeger SL (1986) Longitudinal Data Analysis Using Generalized Linear Models. Biometrica 73, 13-22.

20. Faure H, Preziosi P, Roussel A et al. (2006). Factors influencing blood concentration of retinol, $\alpha$ tocopherol, vitamin C, and $\beta$-carotene in the French participants of the SU.VI.MAX trial. Eur J Clin Nutr 60, 706-717

21. Tatsumi Y, Ishihara J, Morimoto A et al. (2014) Seasonal differences in total antioxidant capacity intake from foods consumed by a Japanese population. Eur J Clin Nutr 68, 799-803

22. Vassallo MF, Banerji A, Rudders S et al. (2010). Season of birth and food allergy in children. Ann Allergy Asthma Immunol 104, 307-313.

23. Koplin, J, Allen K, Gurrin L et al. (2008), Is caesarean delivery associated with sensitization to food allergens and IgE - mediated food allergy: A systematic review. Pediatr Allergy Immunol 19, 682-687

24. Botha M, Basera W, Facey-Thomas He et al. (2019) Rural and urban food allergy prevalence from the South African Food Allergy (SAFFA) study. J Allergy Clin Immunol 143, 662-668

25. Willett W (1998) Nutritional Epidemiology. 2nd ed. ed. New York, NY: Oxford University Press.

26. Tuokkola J, Luukkainen P, Tapanainen H et al. (2016). Maternal diet during pregnancy and lactation and cow's milk allergy in offspring. Eur J Clin Nutr 70, 554-559 .

27. Vaarala O, Atkinson MA, Neu J (2008) The "perfect storm" for type 1 diabetes: the complex interplay between intestinal microbiota, gut permeability, and mucosal immunity. Diabetes 5, 2555-2562.

28. West C., Dunstan J., McCarthy S et al (2012) Association between maternal antioxidant intakes in pregnancy and infant allergic outcomes. Nutrients 11, 1747-1758.

29. Litonjua AA (2012) Fat-soluble vitamins and atopic disease: what is the evidence? Proc Nutr Soc 71, 67-74.

30. Gostner J, Becker K, Ueberall F et al. (2015) The good and bad of antioxidant foods: An immunological perspective. Food Chem Toxicol 80, 72-79

31. Devereux G, McNeill G, Newman G et al. (2007) Early childhood wheezing symptoms in relation to plasma selenium in pregnant mothers and neonates. Clin Exp Allergy 37, 1000-1008.

32. Shaheen SO, Newson RB, Henderson AJ et al. (2004) Umbilical cord trace elements and minerals and risk of early childhood wheezing and eczema. Eur Respir J 24,, 292-297. 
33. Murr C, Schroecksnadel K, Winkler C et al. (2005) Antioxidants may increase the probability of developing allergic diseases and asthma. Med Hypotheses 64, 973-977.

34. Ruhl R (2007) Effects of dietary retinoids and carotenoids on immune development. Proc Nutr Soc 66, 458-469.

35. Uusitalo L, Uusitalo U, Ovaskainen ML et al. (2008) Sociodemographic and lifestyle characteristics are associated with antioxidant intake and the consumption of their dietary sources during pregnancy. Public Health Nutr 11, 1379-1388.

36. Cook-Mills J. M. (2015). Maternal influences over offspring allergic responses. Curr Allergy and Asthma Rep, 15, 501. 
Table 1. Maternal daily intake of nutrients from diet and supplements (total) and from diet during pregnancy $(\mathrm{N}=4403)$

\begin{tabular}{lcc}
\hline & Mean & SD \\
\hline Vitamin A total $(\mathrm{RAE} \dagger, \mu \mathrm{g})$ & $1362 \cdot 5$ & $832 \cdot 6$ \\
Vitamin A diet $(\mu \mathrm{g})$ & $1344 \cdot 8$ & $826 \cdot 3$ \\
Vitamin C total $(\mathrm{mg})$ & $221 \cdot 2$ & $144 \cdot 5$ \\
Vitamin C diet $(\mathrm{mg})$ & $197 \cdot 8$ & $116 \cdot 3$ \\
Vitamin E total $(\mathrm{mg})$ & $13 \cdot 1$ & $8 \cdot 6$ \\
Vitamin E diet $(\mathrm{mg})$ & $11 \cdot 8$ & $4 \cdot 3$ \\
$\beta$-carotene total $(\mu \mathrm{g})$ & $4479 \cdot 7$ & $3825 \cdot 9$ \\
$\beta$-carotene diet $(\mu \mathrm{g})$ & $4280 \cdot 2$ & $3706 \bullet 0$ \\
Retinol total $(\mu \mathrm{g})$ & $917 \cdot 8$ & $715 \cdot 1$ \\
Retinol diet $(\mu \mathrm{g})$ & $916 \bullet 6$ & $713 \cdot 2$ \\
Selenium total $(\mu \mathrm{g})$ & $91 \cdot 4$ & $29 \cdot 3$ \\
Selenium diet $(\mu \mathrm{g})$ & $84 \cdot 6$ & $25 \cdot 4$ \\
Zinc total $(\mathrm{mg})$ & $18 \cdot 9$ & $6 \cdot 6$ \\
Zinc diet $(\mathrm{mg})$ & $16 \bullet 8$ & $4 \cdot 9$ \\
\hline
\end{tabular}

$†$ Retinol equivalent $(\mathrm{RAE})=1 \mu \mathrm{g}$ of retinol $=12 \mu \mathrm{g}$ of ß-carotene 
Table 2. Distribution of background characteristics of all children who participated in the study and for cow’s milk allergic children (cases)

\begin{tabular}{|c|c|c|c|c|c|c|c|}
\hline & \multirow{2}{*}{\multicolumn{2}{|c|}{$\begin{array}{c}\text { All participants } \\
\mathrm{N}=4403\end{array}$}} & \multirow{2}{*}{\multicolumn{2}{|c|}{$\begin{array}{c}\text { Non-Cases } \\
\mathrm{N}=3994\end{array}$}} & \multicolumn{3}{|c|}{ Cow's milk allergy $\dagger$} \\
\hline & & & & & \multicolumn{2}{|c|}{$\begin{array}{c}\text { Cases } \\
\mathrm{N}=409\end{array}$} & \multirow[b]{2}{*}{ p-value* } \\
\hline & $\%$ & $\mathrm{n}$ & $\%$ & $\mathrm{n}$ & $\%$ & $\mathrm{n}$ & \\
\hline$\overline{S e x}$ & & & & & & & $0 \bullet 001$ \\
\hline Boys & $52 \bullet 9$ & 2329 & $52 \bullet 1$ & 2081 & $60 \bullet 6$ & 248 & \\
\hline Girls & $47 \bullet 1$ & 2074 & $47 \bullet 9$ & 1913 & $39 \bullet 4$ & 161 & \\
\hline Season of birth & & & & & & & $0 \bullet 421$ \\
\hline Spring (April-May) & $18 \bullet 3$ & 806 & $18 \bullet 5$ & 739 & $16 \bullet 1$ & 66 & \\
\hline Summer (June-August) & $26 \bullet 6$ & 1169 & $26 \bullet 7$ & 1065 & $25 \bullet 4$ & 104 & \\
\hline Fall (September-November) & $22 \bullet 5$ & 990 & $22 \bullet 5$ & 898 & $22 \bullet 5$ & 92 & \\
\hline Winter (December-March) & $32 \bullet 7$ & 1439 & $32 \bullet 4$ & 1292 & $35 \bullet 9$ & 147 & \\
\hline Age of the mother at delivery (years) & & & & & & & $0 \bullet 051$ \\
\hline$<25$ & $18 \bullet 4$ & 811 & $18 \bullet 9$ & 755 & $13 \bullet 7$ & 56 & \\
\hline $25-29$ & $35 \bullet 1$ & 1547 & $35 \bullet 0$ & 1398 & $36 \bullet 4$ & 149 & \\
\hline $30-34$ & $29 \bullet 1$ & 1282 & $28 \bullet 7$ & 1148 & $32 \bullet 8$ & 134 & \\
\hline$>35$ & $17 \bullet 3$ & 763 & $17 \bullet 4$ & 693 & $17 \bullet 1$ & 70 & \\
\hline Maternal vocational education & & & & & & & $<0 \bullet 001$ \\
\hline No professional education & $6 \bullet 2$ & 272 & $6 \bullet 3$ & 253 & $4 \bullet 7$ & 19 & \\
\hline Vocational school or course & $27 \bullet 1$ & 1191 & $27 \bullet 9$ & 1116 & $18 \bullet 3$ & 75 & \\
\hline Upper secondary vocational education & $43 \bullet 5$ & 1915 & $42 \bullet 9$ & 1713 & $49 \bullet 4$ & 202 & \\
\hline Academic education & $23 \bullet 3$ & 1025 & $22 \bullet 8$ & 912 & $27 \bullet 6$ & 113 & \\
\hline Maternal smoking status during pregnancy & & & & & & & $0 \bullet 005$ \\
\hline No & $90 \cdot 2$ & 3972 & $89 \bullet 8$ & 3587 & $94 \bullet 1$ & 385 & \\
\hline Yes & $9 \bullet 8$ & 431 & $10 \bullet 2$ & 407 & $5 \bullet 9$ & 24 & \\
\hline The mode of delivery & & & & & & & $0 \bullet 883$ \\
\hline Section & $12 \bullet 2$ & 539 & $12 \bullet 2$ & 488 & $12 \bullet 5$ & 51 & \\
\hline Vaginal & $87 \bullet 8$ & 3864 & $87 \bullet 8$ & 3506 & $87 \bullet 5$ & 358 & \\
\hline
\end{tabular}


Urbanity of the place of living

Rural

$12 \bullet 5 \quad 549$

$9 \bullet 6 \quad 423$

Semi-urban

Urban

Maternal use of vitamin A supplements during pregnancy $\ddagger$

$$
\text { No }
$$

Yes

Maternal use of vitamin C supplements during pregnancy $\ddagger$

$$
\text { No }
$$

Yes

Maternal use of vitamin E supplements during pregnancy

$$
\text { No }
$$

Yes

Maternal asthma or allergic rhinitis§

$$
\text { No }
$$

Yes

Missing information

Paternal asthma or allergic rhinitis§

$$
\text { No }
$$

Yes

Missing information

Pets inside home during the first year of life§

$$
\text { No }
$$

Yes

Missing information

$78 \bullet 0$

3431

$93 \bullet 7 \quad 4124$

$6 \bullet 3$

279

$32 \bullet 2 \quad 1417$

$34 \bullet 7 \quad 1529$

54•1 1331

$45 \bullet 9 \quad 1130$

1942

$60 \bullet 5 \quad 1466$

$39 \bullet 5$

956
1981

$67 \bullet 7 \quad 1720$

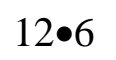

505

$9 \bullet 6 \quad 382$

$77 \bullet 8 \quad 3107$

$10 \bullet 8$

$10 \bullet 0$

$79 \bullet 2$

44

41

324

$0 \bullet 032$

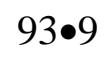

3751

$91 \bullet 2$

373

$6 \bullet 1$

243

$8 \bullet 8$

36

$0 \bullet 007$

$\begin{array}{llllll}67 \bullet 8 & 2986 & 68 \bullet 4 & 2733 & 61 \bullet 9 & 253\end{array}$

$31 \bullet 6$

1261

$38 \bullet 1 \quad 156$

$0 \bullet 001$

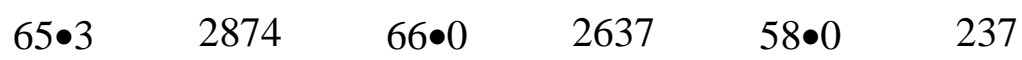

$34 \bullet 7 \quad 1529 \quad 34 \bullet 0 \quad 1357 \quad 42 \bullet 1 \quad 172$

$34 \bullet 0 \quad 1357$

$55 \bullet 3 \quad 1224$

$43 \bullet 2 \quad 107$

$44 \cdot 7 \quad 989$

$56 \bullet 9$

141

1781

$61 \bullet 7 \quad 1343$

$38 \bullet 3$

835

$50 \bullet 4-123$

1816

$49 \bullet 6$

$<0 \bullet 001$

$66 \bullet 9 \quad 1530$

1530
757

33•1 757

1707

$74 \bullet 8$

$25 \bullet 2$

$0 \bullet 011$

* Comparison with $\chi^{2}$-test comparing distributions of cow's milk allergy across the categories.

$\dagger$ Cumulative incidence of cow's milk allergy by the age of 3 years

$\ddagger$ Including the use of multivitamin supplements

$\S$ Information was collected from the asthma and allergy study, which was performed at the child's age of 5 years 
Table 3. The risk of cow's milk allergy in the offspring by 3 years of age associated with maternal daily intake of energy and nutrients during pregnancy. Both total intake and intake from food sources alone are reported for those nutrients which are also derived from supplements.

\begin{tabular}{|c|c|c|c|c|}
\hline & \multicolumn{2}{|c|}{$\begin{array}{c}\text { Unadjusted } \\
\mathrm{n}=409 \dagger / 4403 \ddagger\end{array}$} & \multicolumn{2}{|c|}{$\begin{array}{c}\text { Adjusted analysis§ } \\
\mathrm{n}=409 \dagger / 4403 \ddagger\end{array}$} \\
\hline & $\mathrm{OR} \|$ & $95 \%$ CI & OR $\|$ & $95 \% \mathrm{CI}$ \\
\hline Energy & $0 \bullet 97$ & $0 \bullet 88-1 \bullet 08$ & $0 \bullet 98$ & $0 \bullet 88-1 \bullet 09$ \\
\hline Fat & $0 \cdot 93$ & $0 \bullet 83-1 \bullet 03$ & $0 \cdot 95$ & $0 \bullet 85-1 \bullet 07$ \\
\hline Protein & $0 \bullet 97$ & $0 \bullet 88-1 \bullet 08$ & $0 \cdot 94$ & $0 \bullet 84-1 \bullet 04$ \\
\hline Carbohydrates & $1 \bullet 07$ & $0 \cdot 96-1 \cdot 19$ & $1 \bullet 07$ & $0 \cdot 95-1 \cdot 20$ \\
\hline Vitamin A total & $1 \cdot 02$ & $0 \cdot 93-1 \cdot 11$ & $1 \cdot 02$ & $0 \cdot 93-1 \cdot 12$ \\
\hline Vitamin A diet & $1 \bullet 01$ & $0 \cdot 93-1 \cdot 11$ & $1 \cdot 02$ & $0 \cdot 93-1 \cdot 12$ \\
\hline Vitamin C total & $1 \bullet 07$ & $0 \cdot 97-1 \cdot 17$ & $1 \cdot 06$ & $0 \cdot 95-1 \cdot 17$ \\
\hline Vitamin C diet & $1 \bullet 03$ & $0 \cdot 93-1 \cdot 14$ & $1 \cdot 02$ & $0 \cdot 91-1 \cdot 14$ \\
\hline Vitamin E total & $1 \cdot 04$ & $0 • 99-1 \bullet 09$ & $1 \cdot 03$ & $0 \cdot 98-1 \bullet 08$ \\
\hline Vitamin E diet & $1 \cdot 11$ & $1 \cdot 00-1 \cdot 22$ & $1 \bullet 06$ & $0 \cdot 96-1 \cdot 18$ \\
\hline$\beta$-carotene total & $1 \cdot 12$ & $1 \cdot 03-1 \cdot 21$ & $1 \cdot 10$ & $1 \cdot 02-1 \cdot 20$ \\
\hline$\beta$-carotene diet & $1 \cdot 11$ & $1 \cdot 03-1 \cdot 20$ & $1 \cdot 10$ & $1 \cdot 01-1 \cdot 19$ \\
\hline Retinol total & $0 \bullet 93$ & $0 \bullet 84-1 \bullet 04$ & $0 \cdot 95$ & $0 \bullet 85-1 \bullet 06$ \\
\hline Retinol diet & $0 \cdot 94$ & $0 \bullet 84-1 \bullet 04$ & $0 \cdot 95$ & $0 \bullet 85-1 \bullet 06$ \\
\hline Selenium total & $1 \cdot 03$ & $0 \cdot 94-1 \cdot 14$ & $1 \bullet 01$ & $0 \cdot 91-1 \cdot 11$ \\
\hline Selenium diet & $0 \cdot 94$ & $0 \bullet 85-1 \cdot 04$ & $0 \cdot 93$ & $0 \cdot 84-1 \bullet 03$ \\
\hline Zinc total & $1 \bullet 09$ & $0 \bullet 99-1 \bullet 19$ & $1 \cdot 06$ & $0 \cdot 96-1 \cdot 16$ \\
\hline Zinc diet & $1 \cdot 02$ & $0 \cdot 92-1 \cdot 13$ & $0 \bullet 99$ & $0 \cdot 89-1 \cdot 11$ \\
\hline
\end{tabular}

$\dagger$ Number of children with cow’s milk allergy

$\ddagger$ Number of children in the analysis

$\S$ Adjusted for study center, sex, birth weight of the child, maternal age and education, maternal smoking during pregnancy, duration of gestation, mode of delivery, number of older siblings, season of birth, urbanity of living environment, and length of breastfeeding.

|| Odds ratios are presented per 1 standard deviation increment of the particular nutrient intake 
Figure 1 The flow chart of the pregnancy cohort within the DIPP nutrition study

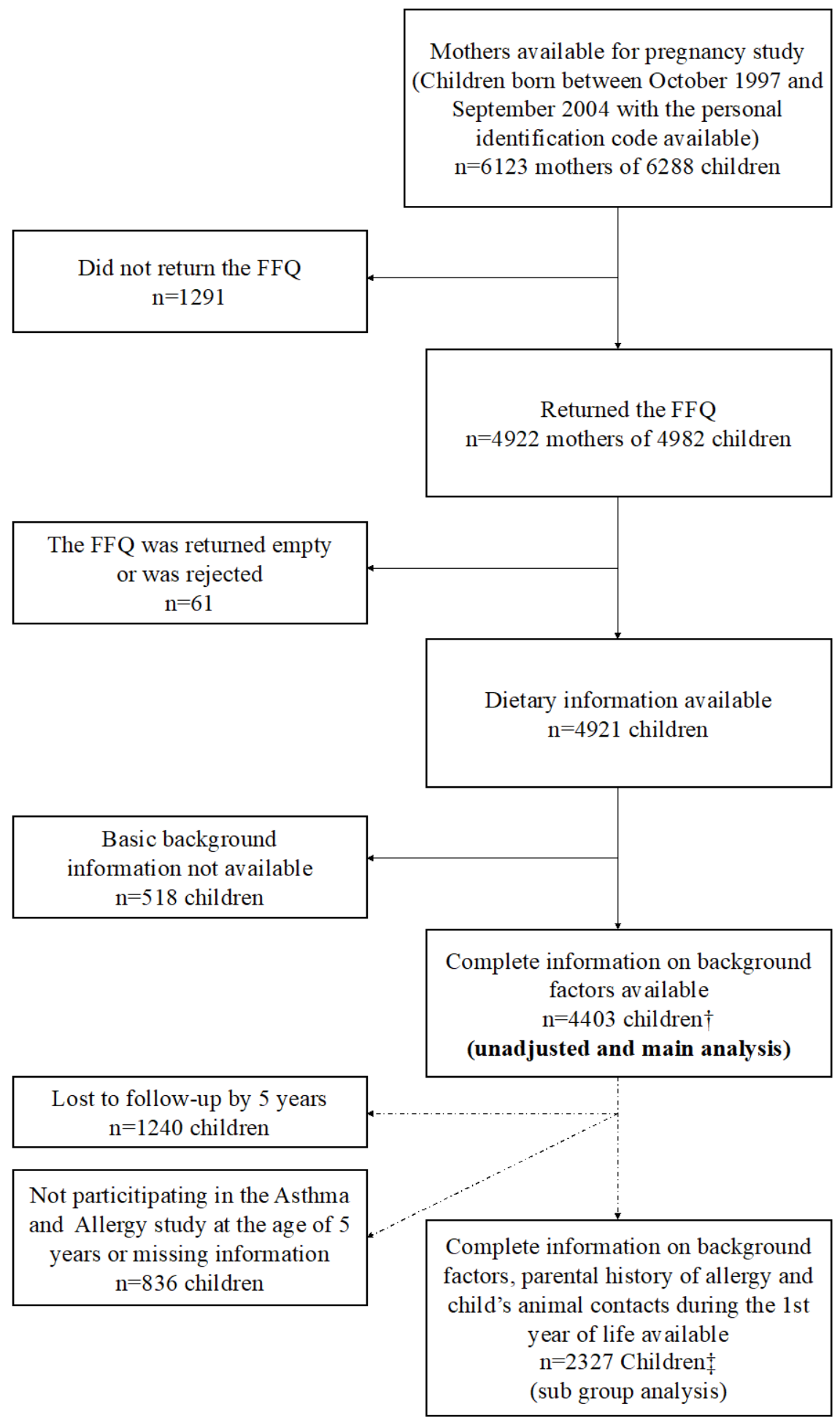

FFQ, food frequency questionnaire

†The information about the study center, gender, season of birth, birth weight of the child, maternal age and education, maternal smoking during pregnancy, duration of gestation, mode of delivery, number of older siblings, urbanity of living environment, and length of breastfeeding were included

$\ddagger$ The information about the parental allergic rhinitis and asthma, child’s visits to a stable, and pet keeping during the first year of life were included 
Supplementary 1. The risk of cow's milk allergy in the offspring by 3 years of age associated with maternal daily intake of energy and energyadjusted nutrients during pregnancy in a sub group of children participating both in maternal diet and Asthma and Allergy studies, and having all the background data available $(\mathrm{n}=2327)$. Both total intake and intake from food sources alone are reported for those nutrients which are also derived from supplements.

\begin{tabular}{|c|c|c|c|c|c|c|}
\hline & \multicolumn{2}{|c|}{$\begin{array}{c}\text { Unadjusted } \\
\mathrm{n}=234 \dagger / 2327 \ddagger\end{array}$} & \multicolumn{2}{|c|}{$\begin{array}{c}\text { Adjusted analysis } 1 \S \\
\mathrm{n}=234 \dagger / 2327 \ddagger\end{array}$} & \multicolumn{2}{|c|}{$\begin{array}{c}\text { Adjusted analysis } 2 \| \\
\mathrm{n}=234 \dagger / 2327 \ddagger\end{array}$} \\
\hline & ORף & $95 \%$ CI & ORף & $95 \% \mathrm{CI}$ & ORף & $95 \%$ CI \\
\hline Energy & $0 \bullet 89$ & $0 \cdot 77-1 \cdot 02$ & $0 \bullet 87$ & $0 \cdot 76-1 \cdot 01$ & $0 \bullet 86$ & $0 \cdot 74-1 \cdot 00$ \\
\hline Fat & $0 \bullet 96$ & $0 \cdot 82-1 \cdot 12$ & $0 \cdot 94$ & $0 \bullet 80-1 \cdot 10$ & $0 \cdot 93$ & $0 \cdot 79-1 \cdot 10$ \\
\hline Protein & $0 \cdot 91$ & $0 \bullet 80-1 \bullet 04$ & $0 \bullet 90$ & $0 \cdot 78-1 \cdot 02$ & $0 \bullet 89$ & $0 \cdot 78-1 \cdot 02$ \\
\hline Carbohydrates & $1 \bullet 06$ & $0 \cdot 91-1 \cdot 24$ & $1 \cdot 10$ & $0 \cdot 93-1 \cdot 28$ & $1 \cdot 10$ & $0 \cdot 93-1 \cdot 29$ \\
\hline Vitamin A total & $1 \cdot 04$ & $0 \cdot 92-1 \cdot 17$ & $1 \cdot 02$ & $0 \cdot 90-1 \cdot 16$ & $1 \cdot 03$ & $0 \cdot 90-1 \cdot 17$ \\
\hline Vitamin A diet & $1 \cdot 03$ & $0 \bullet 91-1 \cdot 16$ & $1 \bullet 01$ & $0 \bullet 89-1 \cdot 15$ & $1 \bullet 01$ & $0 \cdot 89-1 \cdot 16$ \\
\hline Vitamin C total & $1 \cdot 07$ & $0 \cdot 95-1 \cdot 20$ & $1 \bullet 05$ & $0 \cdot 93-1 \cdot 19$ & $1 \cdot 06$ & $0 \cdot 93-1 \cdot 19$ \\
\hline Vitamin C diet & $1 \bullet 05$ & $0 \cdot 91-1 \cdot 20$ & $1 \cdot 04$ & $0 \cdot 90-1 \cdot 19$ & $1 \bullet 04$ & $0 \cdot 90-1 \cdot 21$ \\
\hline Vitamin E total & $1 \bullet 04$ & $0 \cdot 97-1 \cdot 11$ & $1 \cdot 03$ & $0 \cdot 96-1 \bullet 11$ & $1 \bullet 04$ & $0 \cdot 98-1 \cdot 10$ \\
\hline Vitamin E diet & $1 \bullet 11$ & $0 \cdot 97-1 \cdot 27$ & $1 \cdot 07$ & $0 \cdot 92-1 \cdot 24$ & $1 \bullet 06$ & $0 \cdot 91-1 \cdot 23$ \\
\hline$\beta$-carotene total & $1 \cdot 14$ & $1 \cdot 02-1 \cdot 26$ & $1 \cdot 14$ & $1 \cdot 02-1 \cdot 27$ & $1 \cdot 12$ & $1 \cdot 00-1 \cdot 26$ \\
\hline$\beta$-carotene diet & $1 \bullet 11$ & $1 \cdot 00-1 \cdot 23$ & $1 \cdot 11$ & $0 \cdot 99-1 \cdot 24$ & $1 \bullet 09$ & $0 \cdot 97-1 \cdot 23$ \\
\hline Retinol total & $0 \cdot 96$ & $0 \cdot 83-1 \cdot 10$ & $0 \cdot 94$ & $0 \bullet 81-1 \bullet 09$ & $0 \cdot 95$ & $0 \cdot 82-1 \cdot 10$ \\
\hline Retinol diet & $0 \bullet 96$ & $0 \bullet 84-1 \cdot 10$ & $0 \cdot 94$ & $0 \bullet 82-1 \cdot 09$ & $0 \cdot 96$ & $0 \bullet 82-1 \cdot 11$ \\
\hline Selenium total & $1 \bullet 04$ & $0 \cdot 90-1 \cdot 18$ & $1 \cdot 01$ & $0 \bullet 87-1 \bullet 16$ & $1 \cdot 00$ & $0 \cdot 87-1 \cdot 15$ \\
\hline Selenium diet & $0 \bullet 88$ & $0 \cdot 77-1 \bullet 01$ & $0 \bullet 85$ & $0 \cdot 74-0 \cdot 98$ & $0 \bullet 85$ & $0 \cdot 74-0 \cdot 98$ \\
\hline Zinc total & $1 \cdot 15$ & $1 \cdot 02-1 \cdot 30$ & $1 \cdot 14$ & $1 \cdot 00-1 \cdot 29$ & $1 \cdot 13$ & $1 \cdot 00-1 \cdot 27$ \\
\hline Zinc diet & $1 \cdot 04$ & $0 \cdot 91-1 \cdot 19$ & $1 \cdot 04$ & $0 \cdot 90-1 \cdot 19$ & $1 \cdot 04$ & $0 \cdot 90-1 \cdot 19$ \\
\hline
\end{tabular}


$\dagger$ Number of children with cow’s milk allergy

$\ddagger$ Number of children in the analysis

§Adjusted for study center, sex, birth weight of the child, maternal age and education, maternal smoking during pregnancy, duration of gestation, mode of delivery, number of older siblings, season of birth, urbanity of living environment, and length of breastfeeding.

\|Adjusted additionally for parental allergic rhinitis and asthma, child's visits to a stable and pet keeping during the first year of life. This additional information was collected when the child was 5 years old.

ๆ Odds ratios are presented per 1 standard deviation increment of the particular nutrient intake 